$\mathbb{T}$ periodica polytechnica

Civil Engineering

$55 / 2$ (2011) 99,106

doi: 10.3311/pp.ci.2011-2.02

web: http://www.pp.bme.hu/ci

(c) Periodica Polytechnica 2011

RESEARCH ARTICLE

\section{Application of numerical integration techniques for orbit determination of state-of-the-art LEO satellites}

\author{
Balázs Somodi / Lóránt Földváry
}

Received 2011-02-25, accepted 2011-05-17

\begin{abstract}
Classical numerical integration methods have been tested for determining the orbit of most recent Low Earth Orbiter (hereafter LEO) satellites. In general, numerical integration techniques for orbit determination are commonly used to fill the gap between two discrete, observed epochs. In this study orbits have been determined using the EGM96 gravity model by the Euler, Runge-Kutta, Bulirsch-Stoer and Adams-Moulton numerical integration techniques among others. This analysis is performed for a LEO, the GOCE, and for medium altitude satellite, one GPS satellite. The orbits are integrated under different assumptions on the roughness of the force model, considering effects of the ellipticity, high order gravity and non-static Earth generated accelerations on the orbit.
\end{abstract}

\section{Keywords}

orbit integration $\cdot$ LEO $\cdot$ ellipticity $\cdot$ high order gravity $\cdot$ tides . air drag

\section{Acknowledgement}

This work is connected to the scientific program of the "Development of quality-oriented and harmonized $R+D+I$ strategy and functional model at BME" project. This project is supported by the New Hungary Development Plan (Project ID: TÁMOP4.2.1/B-09/1/KMR-2010-0002).

\section{Balázs Somodi}

Faculty of Civil Engineering, Budapest University of Technology and Economics, H-1111 Budapest Múegyetem rkp 3, H-1111 Budapest, Hungary

\section{Lóránt Földváry}

Dept. of Geodesy and Surveying, Physical Geodesy and Geodesical Research Group of the HAS, Budapest University of Technology and Economics, Múegyetem rkp 3, H-1111 Budapest, Hungary

e-mail: fl@sci.fgt.bme.hu

\section{Introduction}

There are countless reasons why orbit determination of a celestial body with the use of numerical integration techniques can be a demand. In many cases of the classical astronomical applications, these techniques are applied in order to forecast the motion of a celestial body for a long period, such as weeks, months or even years.

In this study we deal with a specific need of orbit determination that is for satellite geodesy. Satellites are the key instruments of geodesy, which are used for several applications including navigation, positioning, altimetry and Earth observation [23]. For many applications of satellite geodesy, low altitudes are preferred in order to get better spatial resolution, especially for Earth observing tasks, which are recently in the forefront of geodetic researches. These satellites at very low altitude, generally below $2000 \mathrm{~km}$ are the so-called Low Earth Orbiters (LEO). Meanwhile, the low altitude is a barrier for the time duration of the mission. The altitude is always chosen to be an optimal balance between these two requirements. Due to the technical developments, gradually the feasible altitude became notably lower, and already reached $250-350 \mathrm{~km}$ for the present satellites (Fig. 1). The figure shows the altitude of geodetic LEOs as a function of the date of their launch. The date of launch has been chosen for referencing in time according to an implicite assumption that the altitude of any missions was based on the technical limitations before the date of launch. The figure is based on the altitude of the following 62 satellites: ECHO I, TRANSIT satellites (OSCAR), ANNA 1B, EXPLORER-19, ECHO II, BEACON EXPLORER-B, GEOS-1, EXPLORER-39, GEOS-2, STARLETTE, GEOS-3, SEASAT-1, GEOSAT, AJISAI, SPOT1, MOS-1, SPOT-2, ERS-1, TOPEX-POSSEIDON, SPOT3, ERS-2, RADARSAT-1, SeaStar, TRMM, SPOT-4, Landsat 7, QuikSCAT, ACRIMSAT, CBERS (1, 2, 2B), IKONOS, CHAMP, EO-1, Jason-1, QuickBird, Envisat, GRACE, Aqua, SPOT-5, ICESat, SORCE, Aura, PARASOL, Monitor-E, EROS A, ALOS, Arirang-2, CALIPSO, CloudSAT, COSMIC, EROS B, TerraSAR-X, RADARSAT-2, Jason-2, THEOS, GOCE, SMOS, Proba-2, MTM, Cryosat-2, Aeolus, Swarm and Aquarius, based on [20] and on official internet sites for the recent 
missions. In case of high eccentricity, the mean orbit, i. e. the average of the semi-major and semi-minor axes, is displayed. In case of global satellite systems, the date of launch of the very first satellite was indicated, assuming that the system's scenario was constructed on the level of technololgy of that time. The figure indicates that by mid-90s altitudes below $500 \mathrm{~km}$ become routinely applicable due to the technical developments. The message of the figure is that by now it is inevitable to analize orbit determination related issues at altitudes such as $250-300 \mathrm{~km}$. Generally, the air drag effect at this altitude differs slightly from that at $500 \mathrm{~km}$, however, the effect of the small scale gravity features has an increased role on shaping the orbit of the satellites.

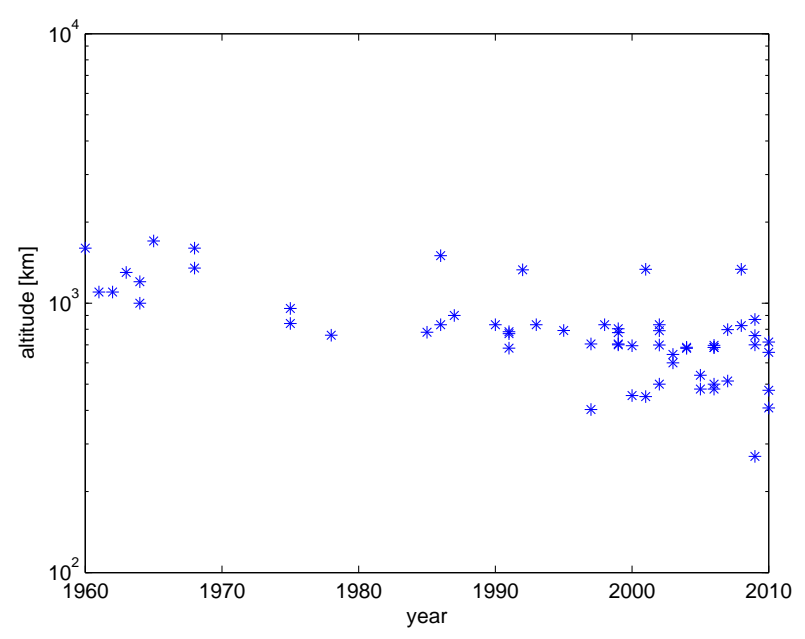

Fig. 1. The altitude of several geodetic LEO satellites as a function of the date of launch

At the dawn of the satellite geodesy, the tracking of the satellites could be done from terrestrial stations only, photographically, with Satellite Laser Ranging or with Doppler [20]. Due to the development of the GPS satellite system with their $20200 \mathrm{~km}$ high altitude, a unique opportunity has been emerged for continuous satellite tracking of LEOs. It made feasible a plenty of demanded scenarios, resulting in a 'new era' of satellite geodesy. Most of the relevant post-GPS LEOs are equipped with a GPSreceiver onboard, which enables a quasi-continuous tracking (sampling the orbit with 1-2 seconds) with an accuracy of 2$3 \mathrm{~cm}$ [22]. According to this, the relevance of orbit integration for long arcs has been decreased. Instead, present applications need orbit integration for periods ranging from some minutes to some hours. These are meant either to fill data gaps (some hours intervals), or determining reduced-dynamic orbit from observed geometric positions (some minutes). In this study we investigate the feasibility of classical numerical integration techniques for the determination of short arcs of LEO orbits. As a case study of a LEO, the satellite on the lowest altitude so far, the GOCE is analyzed [4,6]. In order to provide a base for comparison, the considerably higher orbit of a GPS satellite [20] is also determined with these techniques.

Several numerical integration techniques has already been ap- plied for astronomic applications, such as manifold correction methods for long-term numerical integration of the solar system [8], recurrent power series for orbit determination around an oblate spheriod [9], conservative numerical integration for Henon-Heiles Systems [16], but has never been applied for LEOs. A conceptual difference of abovementioned astronomical applications and LEO orbit determination is that the later is generally a dissipative system with a quite variable force field in time and space. At the present stage of the research none of these methods has been tested, but going to be considered in the future.

The present study deals with those kind of numerical integration techniques, which are classically applied for orbit determination in geodesy [20,23], enlisted in Section 2.2. Application of these techniques for orbit integration of Medium Earth Orbiters (i.e. above $2000 \mathrm{~km}$ and below the geostationary orbits) has a long history [3, 19, 24], though the conceptual difference of a LEO altitude at $500 \mathrm{~km}$ or so, is still a challenge. A similar investigation to the present study has been performed by [5]. That study estimates orbit integration errors of a LEO at an altitude of $400 \mathrm{~km}$ using Runge-Kutta-Fehlberg and AdamsMoulton Predictor-Corrector methods. In the present study similar methods to that two and further four methods have been investigated, and a lower altitude for the LEO has been chosen according to the state-of-the-art techniques.

The orbit integration process is contaminated at least by two error sources: gravity field model errors and numerical integration errors (the later includes the initial state errors). [11] made estimate of satellite orbit error due to gravity field model errors for LEOs on a nearly circular orbit at $400 \mathrm{~km}$ and $800 \mathrm{~km}$ using Kaula's linear perturbation theory [13]. They have found that the RMS orbit error due to the EGM96 model errors is about $0.5 \mathrm{~m}$ and $65 \mathrm{~m}$ when integrating one period meaning one day (at $400 \mathrm{~km})$ and three days $(800 \mathrm{~km})$, respectively. This study employs the same gravity model, but up to a higher maximal degree, for shorter period and for a lower altitude. According to that, in our case gravity field model errors can be in the order of magnitude of some $10 \mathrm{cms}$ at the end of the integration period. Instead of providing a full error analysis of orbit determination, this study purely concentrates on errors of the numerical integration methods, so no estimate on the accuracy of the final coordinates is determined.

\section{Methodology - Basic equations for orbit integration}

\subsection{The differential equation}

Generally, the acceleration of a satellite is determined by considering all the accelerations acting on the satellite (Eq. (1)).

$$
\ddot{r}=a_{E a r t h}+a_{l s}+a_{i n d}+\sum a_{n c}
$$

In Eq. (1) $\ddot{r}$ is the acceleration of the satellite, which is the input information of the orbit determination. The first term on the right-hand side, $a_{\text {Earth }}$ is the gravitational acceleration induced 
by the Earth, which is the largest influence on the satellite. The term $a_{l s}$ and $a_{\text {ind }}$ stands for the direct and indirect effect of the lunisolar gravitation. The last term, $\sum a_{n c}$ contains all the effects of non-gravitational accelerations, such as solar radiation pressure or atmospheric drag.

The actual acceleration vector is preferred to be provided in inertial coordinate system. However, all terms on the right-hand side of Eq. (1) can conveniently be determined in an Earth-fixed one. For example, the gravitational acceleration of the Earth is conventionally $-\operatorname{grad}(V)$, where the potential, $V$, is defined by a spherical harmonic expansion in Earth-fixed system. According this need, Eq. (1) has been modified to

$$
\begin{aligned}
\ddot{r}= & \operatorname{Rot}\left\{-\operatorname{grad}(V)+a_{l s}+a_{i n d}+\sum a_{n c}\right. \\
& +\omega \times(\omega \times R)+2(\omega \times \dot{R})+(\dot{\omega} \times R)\}
\end{aligned}
$$

In Eq. (2) $r$ refers to the inertial and $R$ to the Earth-fixed position vector, and Rot to the rotation matrix between them, i.e. $r=\operatorname{Rot}\{R\}$. The rotation involves apparent accelerations to the equation. These are the last three terms of the right-hand side, which are the centrifugal, Coriolis and Euler accelerations, respectively (for more details see [7]).

Acceleration of the satellite thus can be determined by Eq. (2) at any position $(R, \dot{R})$ in the knowledge of the Earth's gravity field, $V$, and rotation, $\omega$, and the direct and indirect effect of the lunisolar acceleration, $a_{l s}$ and $a_{i n d}$. The gravitational acceleration, $-\operatorname{grad}(V)$, has been computed by spherical harmonic analysis of the EGM96 gravity model [?Lem], up to degree and order 360 . The lunisolar acceleration was modelled by arbitrarily timing the simulation to 01.01.2000, and the actual positions of the Sun, Moon, Jupiter, Mars, Venus and Saturn starting at that epoch has been taken into account. The arbitrary timing should not introduce any error or unlike assumption, since the lunisolar effect does not change relevantly by shifting the test period by some years or decades. Similarly, $a_{i n d}$ has been taken into account for the same time span. It consists of three components, that is the solid Earth tide, ocean tide and polar tide. For the ocean tide a combination of a TOPEX/Poseidon-based and the Schwiderski tide models was used; for solid Earth tide and for polar tide the IERS data and conventions were used [15]. Also, for the angular velocity of the Earth, $\omega$, IERS data was used. Finally, $\sum a_{n c}$ is a very case sensitive variable, and cannot be appropriately simulated, though there are convincing results for modelling it (e.g. [12]). This term has been excluded from the investigation. Generally, exclusion of nongravitational accelerations from Eq. (2) doesn't change drastically the acceleration of the satellite at a discrete epoch. In the case of GRACE (altitude: $500 \mathrm{~km}$ ), the magnitude of the nongravitational accelerations were measured to be in the range of $10^{-8} \mathrm{~m} / \mathrm{s}^{2}$, while that of the gravitational potential is about 8.43 $\mathrm{m} / \mathrm{s}^{2}$ [17]. Thus the non-conservative acceleration is 8 orders of magnitude smaller than the signal, so it is negligible in a single epoch. Nevertheless on a long run it has a notable effect on a
LEO orbit due to the dissipative nature of these accelerations. These have always the same sign, which is cumulated in time, e.g. air drag always slows down a satellite's motion and never gives a push. According to this, for simulation of short-arc orbit integration the air-drag can be neglected. Furthermore, in the case of the GOCE these accelerations are real time compensated by thrusters, so the GOCE orbit literally are not affected by them [1].

In the present study numerical integration techniques are investigated for different accuracy requirements. Accuracy requirements are defined implicitly, described by the refinement of the force model needed for the orbit integration:

1. Applications, for which a spherical Earth approximation is sufficient,

2. Applications, for which an ellipsoidal Earth approximation is demanded,

3. Applications, requiring an exact knowledge of the Earth gravity field,

4. Applications, requiring the consideration of all effects.

For the four different applications, Eq. (2) has been modified according to the description to a spherical approximation (only the spherical part of the term $\operatorname{grad}(V)$ is used, others neglected), to an ellipsoidal approximation (long-wavelength zonals of the term $\operatorname{grad}(V)$ are used, others neglected), to a high order approximation (all terms of $\operatorname{grad}(V)$ are used, others neglected), and to an all-effects-considered approximation (no simplification of the equation), respectively.

\subsection{Numerical integration techniques}

Orbit integration generally is a process of determining position, $r$, in the knowledge of accelerations, $\ddot{r}$, computed by Eq. (2) based on the Hamiltonian mechanics [10]. For using Eq. (2), the knowledge of the position and velocity is required for each step forward. According to that, it is proper to change the position variable to general coordinates, i.e. $x=[r, \dot{r}]$. With that substitution, orbit determination can be simplified to a first order integration:

$$
\dot{x}=\left[\begin{array}{cc}
0 & 1 \\
-\operatorname{grad} V & 0
\end{array}\right] x+\left[\begin{array}{c}
0 \\
a_{l s}+a_{\text {ind }}
\end{array}\right]
$$

For solving Eq. (3) by numerical integration, the following classical techniques have been used:

1. Euler method, which is a first order method

2. Midpoint method, or second order Runge-Kutta method

3. Fourth order Runge-Kutta method

4. Modified midpoint method, which is a high order method with an arbitrary order

5. Bulirsch-Stoer method, a high order method employing the Richardson extrapolation

6. High order polynomial predictor-corrector method

7. Adams-Moulton/Adams-Bashford predictor-corrector method, which is a 4th order linear multistep method 
Tab. 1. Initial coordinates in inertial coordinate system

\begin{tabular}{c|rr}
\hline$[m]$ or $[\mathrm{m} / \mathrm{s}]$ & $G P S$ & GOCE \\
\hline$x$ & 10372205.358 & 6229351.121 \\
$y$ & -13696243.141 & -260596.816 \\
$z$ & 20027165.249 & 2252272.469 \\
$\dot{x}$ & 3565.919 & -2652.114 \\
$\dot{y}$ & 1119.005 & -837.504 \\
$\dot{z}$ & -1111.911 & 7238.332 \\
\hline
\end{tabular}

An overall description of the Euler, Runge-Kutta and predictor-corrector methods is provided by [2]. Bulirsch-Stoer method is described in [18]. For further details on the exact use of them for the present investigation see [21].

\section{Initial Conditions and Parameterization}

For integrating the orbit, initial conditions of the satellite has to be defined by setting the initial position of the satellite, $x=[r, \dot{r}]$ at the beginning of the orbit determination period. This can be equivalently provided by defining six Keplerian elements. For GPS, actual coordinates of a satellite were used. For the GOCE, at the time of this study there were no actual orbits available, thus nominal orbital elements of the GOCE orbit were used, with $251 \mathrm{~km}$ semi-major axis, inclination of 96.6 degree. The real anomaly has been set to $20^{\circ}$, while the rest of the elements, i.e. eccentricity, perigeum and ascending node were set to zero. The altitude of $251 \mathrm{~km}$ for GOCE was set according to the original scenario, which has been modified to $270 \mathrm{~km}$ due to the delay of the launch, which unlikely has resulted in increased Sun activity. The applied initial coordinates are summarized in Table1.

The duration of the integration was chosen to be $10000 \mathrm{~s}$. In the case of GOCE it means less than two revolutions, in the case of GPS it is less than a quarter revolution. The choice of the same duration was defined in order to relate the numerical errors to integration time and not the completed periods.

The step size of the integration was chosen to be $1 \mathrm{~s}$ and $5 \mathrm{~s}$ for GOCE and GPS, respectively. The fine resolution has been chosen in order to include the delicate features of the gravity field variations as much as possible, resulting in a "reliable" orbit. Still, in both cases the precision of the computer and of the used software has not been reached. (The estimated precision of the computation is displayed on those figures later, where it is found to be informative).

The free parameters of the investigated methods have been defined as follows: For the modified midpoint method $4 t h$ and $10 t h$ order chains were tested. For the Bulirsch-Stoer method first the orbit is integrated with some conveniently applicable step sizes, then theoretical infinite step size is achieved by an extrapolation; for the later we have used the Richardson extrapolation. The Adams-Moulton/Adams-Bashford method requires more epochs initially; these have been derived using the $4 t h$ order Runge-Kutta method. The analytical linear predictorcorrector method in our case refers to use a fifth order polyno- mial fitting for both the predictor and the corrector steps.

\section{Results}

\subsection{Computational Time}

The computational time of the methods can be informative from efficiency aspects. These are shown in Table 2 According to our experience, the most time consuming step was the analytical determination of $\dot{x}$ by Eq. (2). In order to confirm it, in Table 2 the number of using Eq. (2) during computation of one step size has also been provided. Since the step size of the GPS is 5 times of that of the GOCE, the computational time of the GOCE orbit took roughly about 5 times of the GPS (cf. last two columns of the table).

\subsection{Accuracy of the methods}

First a rough test of the integrated orbits has been performed: the period of the GOCE orbits derived by different techniques has been determined. In the case of the Euler method it was $5602.5 \mathrm{~s}$, while in the case of all other methods it was $5545.4 \mathrm{~s}$. It suggests that the Euler orbit parts away from the others.

For visualizing the differences of the orbits, the $5 t h$ order predictor-corrector method was chosen to be the reference of comparison. According to the prelavent use of this method, it is presumably assumed to provide a "reliable" estimate, thus differences to the orbit based on that method can be considered as an error estimate to a certain extent. These residuals are shown on Fig. 2 and 3 . In these figures an approximate value for the precision of the computation is also shown, which is based on simple considerations on the limitations of the used hardware and os the software.

Generally, the accuracy of the methods is quite similar for the case of the GPS and of the GOCE, with slightly smaller residuals at the end of the integration period in the case of GPS. This is due to the fact that at the altitude of the GPS satellites the force field is notably smoother and the gravity is smaller. Since the accelerating force is smaller, less numerical error due to its integration occur.

The accuracy of the Euler method is in the same order of magnitude than the coordinates are. The 2 nd order RungeKutta method performs notably better, providing errors below $100 \mathrm{~m}$. The 4th order modified midpoint method manifests an error in the range of $5-10 \mathrm{~m}$, while the $10 \mathrm{th}$ order modified midpoint method performs better with one more order of magnitude. Surprisingly the $4 t h$ order Runge-Kutta and the Bulirsch-Stoer methods agreed completely within the precision of the computer and of the software. The difference of these two methods is also displayed (thin black dotted line), which is under or close to the precision of the computation (thin horizontal solid line). These orbits differ from the reference one for some cm only. Finally, the difference of the two predictor-corrector methods is in range of $\mathrm{mm}$. 
Tab. 2. Computational time of the orbit integration

\begin{tabular}{l|ccc}
\hline & $\begin{array}{r}\text { no. of using } \\
\text { equation } 2\end{array}$ & $\begin{array}{c}\text { runtime for } \\
\text { GPS }[h]\end{array}$ & $\begin{array}{c}\text { runtime for } \\
\text { GOCE }[h]\end{array}$ \\
\hline Euler & 1 & 1.3 & 7.8 \\
2nd order Runge - Kutta & 2 & 3.5 & 11.8 \\
4th order Runge - Kutta & 4 & 7.4 & 26.0 \\
4th order modifiedmidpoint & 4 & 6.8 & 23.5 \\
10th order modified midpoint & 10 & 12.6 & 58.1 \\
Bulirsch - Stoer & 14 & 20.8 & 96.2 \\
5th order polynomial predictor - corrector & 2 & 2.5 & 12.2 \\
Adams - Moulton/Adams - Bashford & 2 & 2.5 & 10.6 \\
\hline
\end{tabular}

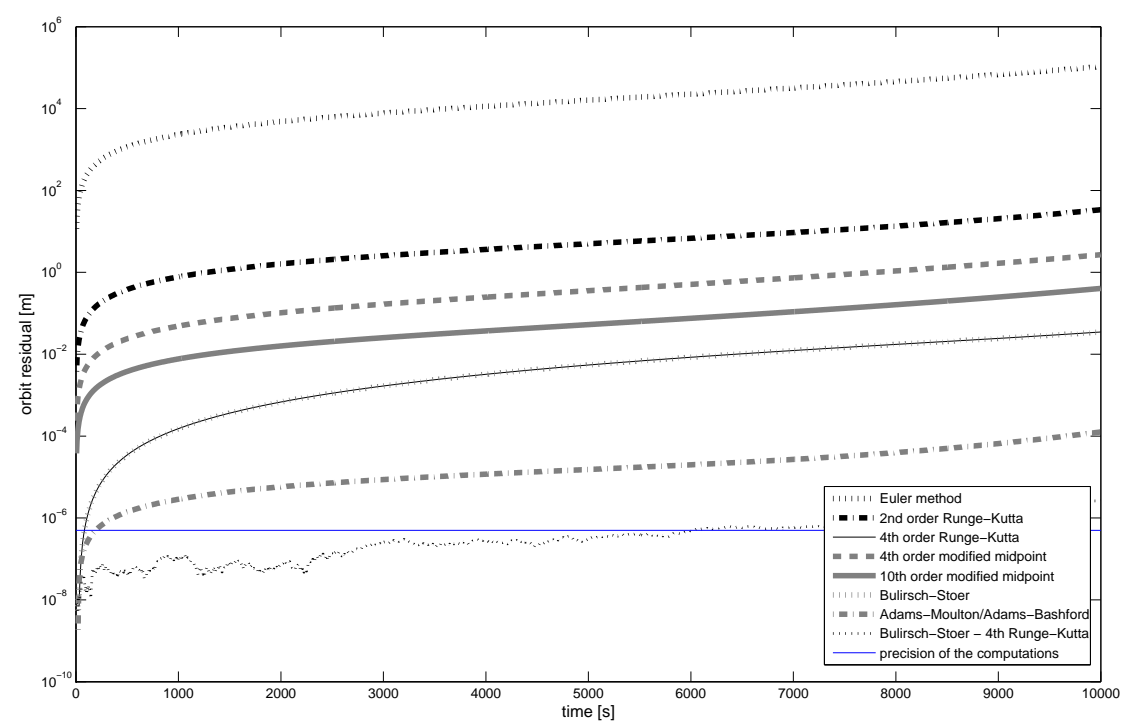

Fig. 2. Residuals to the 5 th order polynomial predictor-corrector orbit for the GPS satellite

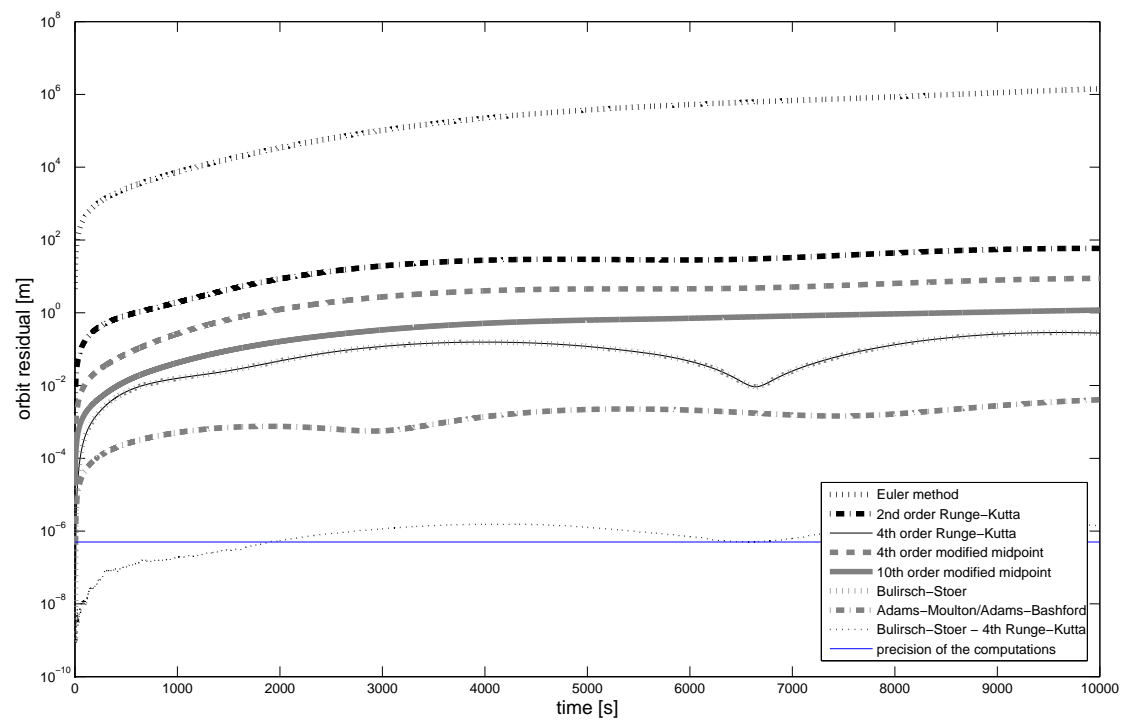

Fig. 3. Residuals to the $5 t h$ order polynomial predictor-corrector orbit for the GOCE satellite 
Tab. 3. Differences to the $5 t h$ order polynomial predictor-corrector-based orbit at the end of the test period, after $10000 \mathrm{~s}$

\begin{tabular}{l|ll}
\hline & $\begin{array}{l}\text { GPS } \\
{[\mathrm{m}]}\end{array}$ & $\begin{array}{l}\text { GOCE } \\
{[\mathrm{m}]}\end{array}$ \\
\hline Euler & $1.062 \cdot 10^{5}$ & $1.429 \cdot 10^{6}$ \\
2nd order Runge - Kutta & $3.386 \cdot 10^{1}$ & $5.874 \cdot 10^{1}$ \\
4th order modified midpoint & $2.703 \cdot 10^{0}$ & $8.838 \cdot 10^{0}$ \\
10th order modified midpoint & $4.059 \cdot 10^{-1}$ & $1.186 \cdot 10^{0}$ \\
4th order Runge - Kutta & $3.497 \cdot 10^{-2}$ & $2.746 \cdot 10^{-1}$ \\
Bulirsch - Stoer & $3.496 \cdot 10^{-2}$ & $2.746 \cdot 10^{-1}$ \\
Adams - Moulton/Adams - Bashford & $1.263 \cdot 10^{-4}$ & $4.063 \cdot 10^{-3}$ \\
\hline
\end{tabular}

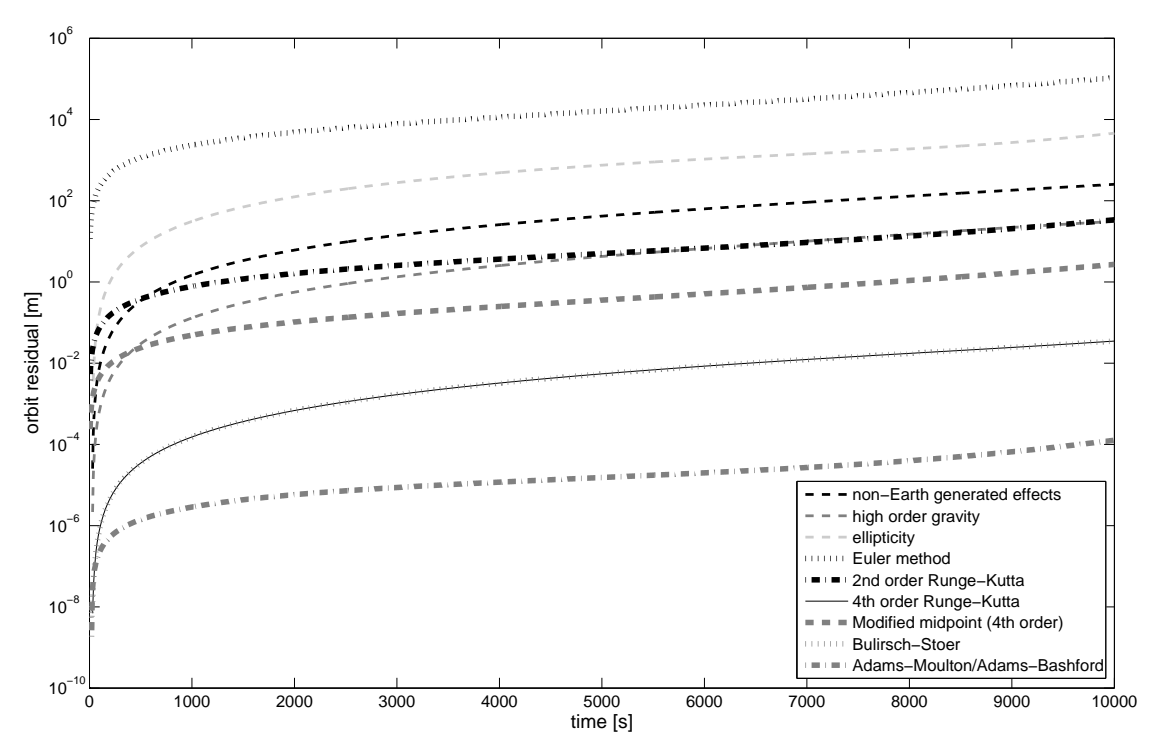

Fig. 4. Effects of the ellipticity, of the high order gravity, and of the non-Earth effects on the GPS orbit along with the residual curves of Fig. 2

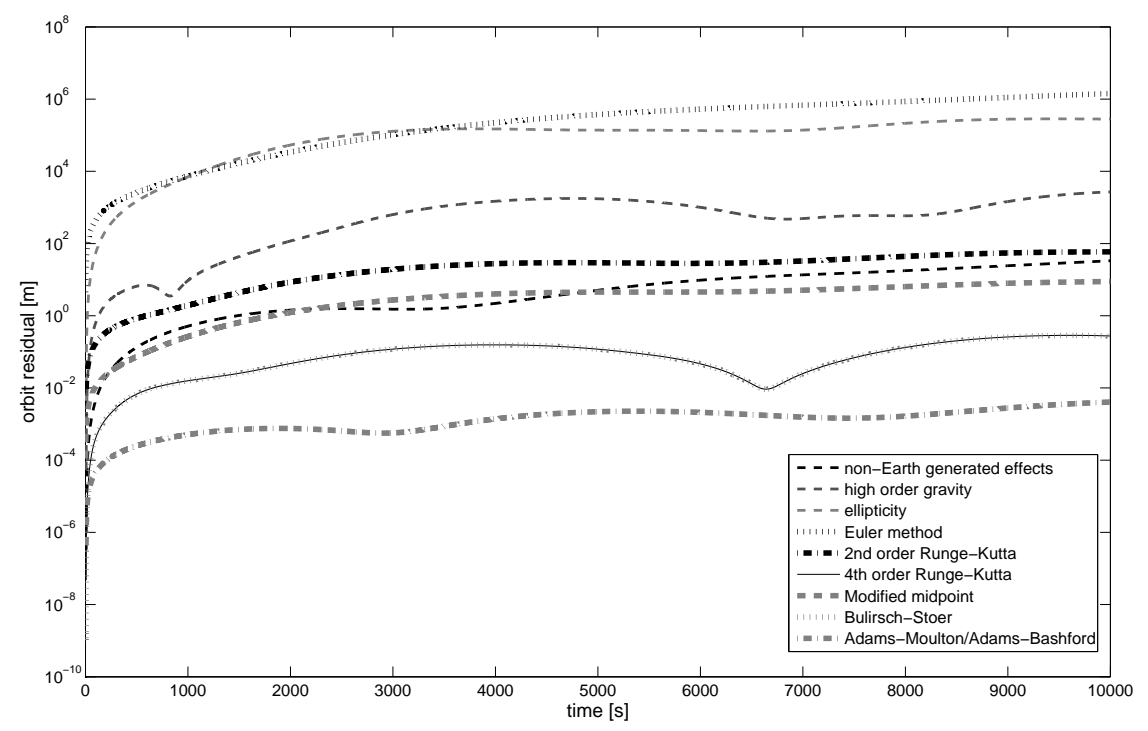

Fig. 5. Effects of the ellipticity, of the high order gravity, and of the non-Earth effects on the GOCE orbit along with the residual curves of Fig.2 
The differences at the end of the period, $10000 \mathrm{~s}$ are displayed in Table 3 Note that in the case of the GOCE it is in some cases not the maximal difference. Since the GOCE completes more than a whole revolution, orbital periodical errors also appear. However, it turned out that the difference from the maximal error due to the periodicity is less than $5 \%$, so makes no essential difference for the conclusion.

\subsection{Components of the force model}

In this section the relation between the accuracy of the methods and the accuracy requirements is investigated: for what accuracy demands the different techniques can be applied efficiently? The orbit integration has been performed with the reference method, the $5 t h$ order polynomial predictor-corrector method under four different assumptions on the force field, as they were defined in Section 2.1 .

The most rough accuracy requirements can be satisfied by assuming a spherical Earth, while a more realistic orbit can be obtained by integrating in an ellipsoidal Earth model. Integrating orbits using these two approximations, the difference of the two orbits describes the effect of the difference of the spherical and the elliptical models on the orbit, that is the ellipticity of the Earth. This is displayed by thin light-grey dashed line. Similarly, difference of orbits integrated in an elliptic Earth and in a high order models (thin grey dashed line), and difference of the high order Earth and the all-effects-considered models (thin black dashed line) have been determined. The first describes the effect of the non-ellipsoidal, or high-order components of the Earth gravity field, while the later estimates the effect of the non-Earth generated accelerations, such as direct and indirect tides on the orbit of the satellite. These are shown on Fig. 4 for GPS and Fig. 5 for GOCE, along the accuracy curves of the orbit integration techniques.

Comparing Fig. 4 and Fig. 5, it is relevant that in both cases after a spherical Earth assumption, the ellipticity has the largest effect on the orbit. However beyond this, in the case of the GOCE, the high order gravity signal has the larger effect on the orbit, while in the case of the GPS it is the non-Earth generated forces. It is in accordance with the task of the satellites: the GOCE is designed to map the high order gravity signal, while for GPS it is rather important to revolve on a stable orbit, so minimal perturbations are preferred.

The interpretation of the accuracy of the numerical integration techniques and of the accuracy requirements on Fig. 4 and 5 is as follows. When the demand is that for a satellite orbit certain signal, e.g. ellipticity should be taken into account, those methods are considered to fulfill the requirements, which lie at least one order of magnitude below the signal curve. According to this, the $10 \mathrm{th}$ order modified midpoint, the $4 \mathrm{th}$ order Runge-Kutta, the Bulirsch-Stoer and the Adams-Moulton / Adams-Bashford methods are found to be generally applicable methods. For as high orbit as that of the GPS, 4th order modified midpoint method should be sufficiently accurate too. Similarly, more refined conclusions can be drawn from the figures, which is provided method by method in the next section.

\section{Conclusions}

In this study the accuracy of numerical integration techniques for orbit determination of geodetic satellites has been investigated. The accuracy estimates are then analyzed from the aspect of the influencing signal, i.e. the force field. The considered components of the force field were the ellipticity of the Earth, the high order gravity information of the Earth and the non-Earth generated effects. The results are summarized for each method one by one in the followings.

Euler method: The simplest and the less accurate numerical integration technique. It causes in a $10000 \mathrm{~s}$ arc errors in the order of magnitude of $10^{6} \mathrm{~m}$ for the case of the GOCE, and $10^{5}$ $\mathrm{m}$ for the GPS. Due to its quick runtime, this method can only be used for robust analysis of very short arcs.

$2 n d$ order Runge-Kutta method: It's runtime is twice of Euler's method's, which means it is still a definitely quick way of orbit determination. It generates errors in the integration of a $10000 \mathrm{~s}$ arc in the range of $10 \mathrm{~m}$. With this limitations, this method can generally be used for robust orbit determination, but it can be appropriate also for integrating longer orbits in a homogeneous ellipsoidal Earth model.

4th order Runge-Kutta and Bulirsch-Stoer methods: These two methods turned out to provide the same results. These are fairly accurate methods, providing at the end of the $10000 \mathrm{~s}$ arc some decimetre errors in the GOCE, and some centimetres in the GPS orbits. These errors are in the range of the gravity field model errors. With this accuracy both methods are applicable for precise orbit determination over short arcs, such as filling of data gaps for some hours, or computing reduced-dynamic orbits with stochastic pulses after each some minutes. For integration of longer arcs, further tests are required. General difference of the two methods is the computational time, which is 3-4 times more for the Bulirsch-Stoer method than that of the $4 t h$ order Runge-Kutta method.

Modified midpoint method: Its efficiency is function of the parameterization. The $4 t h$ order method has quite similar computational time to the $4 t h$ order Runge-Kutta method, but at the end of a $10000 \mathrm{~s}$ long integration, its errors are about 2 orders of magnitude larger than that of the Runge-Kutta method, in the range of some meters. The $10 \mathrm{th}$ order modified midpoint method takes 2-3 times more computational time compared to the $4 t h$ order method, and results in an improvement of an order of magnitude in accuracy. Its use for precise orbit determination is not really suggested, since for LEO orbits the computational errors are in the same order of magnitude than that of the tides (i.e. main components of the non-Earth generated accelerations).

Adams-Moulton/Adams-Bashford and 5th order polynomial predictor-corrector methods: Since the predictor-corrector methods were used in this study as a reference, conclusions on 
accuracy in these cases cannot be drawn. The difference of the two methods to each other is in the sub-millimetre range. It is a kind of validation of the mathematically simpler method, i.e. the Adams-Moulton/Adams-Bashford method, proving that the simplified closed forms of both the prediction and the correction steps can reach the same accuracy than the high order polynomial fitting. A general strength of these methods is the efficient computational time, only 1.5-2 times of that of the Euler method, they take even less time than the $2 n d$ order Runge-Kutta method. Due to the simplicity of the AdamsMoulton/Adams-Bashford method, this is even slightly less. So for precise orbit determination over long arcs these are the most suggested techniques.

All in all, for short arc orbit determination tasks, among the mathematically simple methods, the $4 t h$ order Runge-Kutta method is found to be efficient. For both short and long arc orbits the Adams-Moulton/Adams-Bashford method is the most efficient one among the investigated techniques.

\section{References}

1 GOCE Gravity Field and Ocean Circulation Explorer, Phase A Executive Summary, GOC-RP-AI-0006, Alenia Spazio, 1999.

2 Butcher J C, Numerical methods for ordinary differential equations, John Wiley \& Sons Ltd, 2003.

3 Cojocarua S, A Numerical Approach to GPS Satellite Perturbed Orbit Computation, Journal of Navigation 60 (2007), 483-495, DOI 10.1017/S0373463307004377.

4 Drinkwater M R, Floberghagen R, Haagmans R, Muzi D, Popescu A, GOCE: ESA's first Earth Explorer Core mission, Earth Gravity Field from Space - from Sensors to Earth Sciences (Beutler G B, Drinkwater M, Rummel R, von Steiger R, eds.), Space Sciences Series of ISSI, vol. 18, Kluwer Academic Publishers, Dordrecht, Netherlands, 2003, pp. 419-432, DOI 10.1023/A:1026104216284, (to appear in print).

5 Es-hagh M, Step variable numerical orbit integration of a low earth orbiting satellite, Journal of the Earth \& Space Physics 31 (2005), no. 1, 1-12.

6 Floberghagen R, Drinkwater M, Haagmans R, Kern M, GOCE's Measurements of the Gravity Field and Beyond, ESA Bulletin, ESA, 2008.

7 Földváry L, Bokor Zs, Determination of a CHAMP gravity model based on the Newtonian equation of motion, Periodica Polytechnica Civil Engineering 54 (2010), no. 2, 155-161, DOI 10.3311/pp.ci.2010-2.11.

8 Fukushima T, Efficient Orbit Integration by Orbital Longitude Methods, Proceedings of the Symposium on Celestial Mechanics 36 (2004), 123-129, DOI 10.1086/423042.

9 Hadjifotinou K G, Numerical integration of the variational equations of satellite orbits, Planetary and Space Science 50 (2002), 361-369, DOI 10.1016/S0032-0633(02)00008-9.

10 Hairer E, Norsett S P, Wanner G, Solving ordinary differential equations I: Nonstiff problems, 2nd Edition, Springer Verlag, Berlin, 1993.

11 Hwang C, Hwang L S, Satellite orbit error due to geopotential model error using perturbation theory: applications to ROCSAT-2 and COSMIC missions, Computers \& Geosciences 28 (2002), 357-367, DOI 10.1016/S00983004(01)00053-X.

12 James Raj M X, Sharma R K, Contraction of near-Earth satellite orbits using uniformly regular KS canonical elements in an oblate atmosphere with density scale height variation with altitude, Planetary and Space Science $\mathbf{5 7}$ (2009), 34-41.

13 Kaula W M, Theory of Satellite Geodesy, Blaisdell Publishing Co., London, 1966.
14 Lemoine F G et al., The Development of the Joint NASA GSFC and the National Imagery and Mapping Agency (NIMA) Geopotential Model EGM96, NASA Goddard Space Flight Center, Greenbelt, Maryland, 20771 USA, 1998.

15 McCarthy D D, IERS Conventions, IERS Technical Note, U.S. Naval Observatory, 1996.

16 Minesaki Y, Nakamura Y, A Conservative Numerical Integration Algorithm for Integrable Henon-Heiles System, Proceedings of Institute of Mathematics of NAS of Ukraine, Vol. 50, 2004, pp. 444-449.

17 Paizs Z, Földváry L, Determination of a gravity model based on GRACE microwave ranging observations, Geomatikai Közlemények 10 (2007), 201210.

18 Press W H, Flannery B P, Teukolsky S A, Vetterling W T, Numerical Recipes in C, 2nd Edition, Cambridge University Press, 1988.

19 Rutkowska M, The accuracy of orbit estimation for the low-orbit satellites LARETS and WESTPAC, Advances in Space Research 36 (2005), 498-503, DOI 10.1016/j.asr.2005.04.063.

20 Seeber G, Satellite Geodesy, Walter de Gruyter, Berlin, New York, 1993.

21 Somodi B, Application of numerical integration techniques for orbit determination of geodetic satellites, Scientific Student Work, Budapest Unv. of Techn. and Economics, 2008.

22 Svehla D, Földváry L, From Kinematic Orbit Determination to Derivation of Satellite Velocity and Gravity Field, Observation of the Earth System from Space (Flury J et al., ed.), Springer Verlag, Berlin Heidelberg New York, 2006, pp. 177-192.

23 Torge W, Geodesy, 3rd Edition, Walter de Gruyter, Berlin, New York, 2001. 24 Torrence M H, Dunn P J, Kolenkiewicz R, Charecteristics of the LAGEOS and ETALON Satellites Orbits, Advances in Space Research 16 (1995), (12)21-(12)24, DOI 10.1016/0273-1177(95)98772-G. 